\title{
Ueber die Zerstörung der Hölzer an der Atmosphäre
}

hat Julius Wiesner Beobachtungen und Versuche angestellt und die zum Abschluss gebrachten Theile seiner Untersuchungen, betreffend drei wahrhaft typiscbe Arten der Zerstörung reifer Hölzer, die er mit den Namen "Grauwerden", „Bräunung “ und "staubige Verwesung (staubige Vermoderung)" der Hölzer belegte, veröffentlicht.

I. Das Grauwerden (die Vergraung) des Holzes.

Es giebt eine grosse Menge von Hölzern, die von Laub - und Nadelbäumen herrühren, welche an trockenen Orten im Längsschnitte der Atmosphäre ausgesetzt, in Folge oftmaligen Wechsels von oberflächlicher Befeuchtung durch die atmosphärischen Niederschläge und Austrocknung eine graue Oberflüche annehmen. Diese licht- bis dunkelgraue Schicht des Holzes besteht aus Zellen, welche durch die atmosphärischen Niederschläge ausgelaugt und ihrer Infiltrationsproducte ganz oder zum grossen Theile beraubt wurden, so zwar, dass die zurückbleibenden Membranen bloss aus chemisch-reiner oder nahebei chemisch-reiner Cellulose bestehen. Durch die Volumsänderungen, welche die Zellen beim Feuchtwerden und bei der darauf folgenden Austrocknung erleiden, wird ein grosser Theil der Intercellularsubstanz mechanisch aus dem Holze entfernt, wobei dessen Zellen ganz oder zum Theil isolirt werden. Die vollständig isolirten Zellen fallen vom Holze, welches in Folge der Blosslegung von Zellen eine haarige, manchmal wollige Oberfläche angenommen hat, ab. Die Membranen der unvollständig isolirten Zellen wittern schichtweise - vornehmlich von innen nach aussen $\mathrm{ab}$, gleichzeitig werden die Reste der Zellenwände von bestimmten Puncten aus zerklüftet. Durch diese Klüfte (Tüpfelrisse) kommen die in der Atmosphäre allenthalben vorhandenen Sporen niederer vegetabilischer Organismen (Pilze, Flechten) ins Innere der Zellen. Die auf diese Weise demolirten Zellen des Holzes trennen sich nun nach und nach von dem Holzkörper los, entweder indem sie als solche herausfallen (beinahe alle Markstrahlenzellen, aber nur eine geringe Zahl von Holzzellen) oder indem sie zusammenbrechen. Dieses letztere geschieht gewöhnlich durch allzu starke oder ungleichmässige Abtragung der Zellwand, nicht selten auch unter Mitwirkung von Pilzmycelien, welche mit zunehmender Entwickelung die Zellreste zersprengen. An vielen Holzzellen vergrau- 
ter Hölzer kann man bemerken, dass die an den Membranen sich anklammernden Pilze in Spiralgängen sich gleichsam in die Wand der Zelle zur Hälfte eingraben und nach ihrem Abfallen die Spuren ihrer Wege zuriicklassen.

(H. S ch a cht, in seiner im 3. Bande der Pringsheimschen Jabrbücher erschienenen Abhandlung: über die Veränderung durch Pilze in abgestorbenen Pflanzenzellen belegt diese Spuren mit dem sehr passenden Namen "Pilzbahnen"). Bei allzu reichlicher Entwickelung von Pilzen dringen die Sporen und Mycelien derselben auch in noch unvergraute Zellen ein und rufen dann eine Zerstörungsart des sich hierbei schwärzenden Holzes hervor, welche mit der hier beschriebenen Art der Verwesung nichts gemein hat. Die Holzzellen sind mithin sowohl an Laub-als Nadelhölzern diejenigen Elementarorgane, welche der Atmosphäre bei der Vergrauung an längsten Trotz bieten. Wi es ner untersuchte vollständig isolirte Holzzellen und Markstrahlzellen, erstere von einem vergrauten Weiden-, letztere von einem vergrauten Ahornholze (Acer campestre) herrührend, in welchen noch ganz unverletzte Stärkekörner vorhanden waren.

\section{Die staubige Vermoderung des Holzes.}

Der Hergang bei dieser Verwesung ist folgender: Die an der Atmosphäre liegenden Holzbalken bekommen beim Austrocknen in Folge unregelmässiger Zusammenziehung Längsrisae, die oft ziemlich tief in die Holzmasse eingreifen. In den auf diese Weise entstandenen, nahebei horizontal verlaufenden Spalten sammelt sich das Regenwasser an und bleibt hier in stunden-, oft tagelanger Berührung mit dem Holzkörper, der nun entweder bloss unter dem Einflusse des Wassers, oder was wahrscheinlicher ist, unter dem des Wassers, der Kohlensäure und des Ammoniaks jene chemische und physikalische Umgestaltung erfährt, in Folge deren das Holz beim Druck zwischen den Fingern zu Staub zerfällt. Bis jetzt beobachtete Wiesner dieselbe bloss an Nadelhölzern und zwar bei Weisstanne (Abies pectinata), Fichte (Abies excelsior), Kiefer (Finus sylvestris) und Schwarzföhre (Pinus austriaca).

Das staubigvermoderte $\mathrm{Holz}$ hat eine graugelbe, ochergelbe, hellbraune bis schwarzbraune Farbe; das Pulver der schwarzbraun gewordenen Hölzer ist stets braun. 


\section{Die Zerstörung der Hölzer an der Atmosphäre.}

Alle mikroskopischen Untersuchungen von staubigvermoderten Hölzern ergaben, dass die histologischen Verhältnisse der Elementarorgane bei diesem Vermoderungsacte keine erheblichen Aenderungen erfahren batten; die Membranen der Zellen zeigten keine Aenderung ihrer Structur und auch die Intercellularsubstanz hatte sich vollständig erhalten. Eines war in histologischer Beziehung unverkennbar, nämlich dass die Frühlingslagen des Holzes in der Regel viel schneller diesem Vermoderungsprocesse unterliegen, als die Spätlagen (Sommer- und Herbstlagen) desselben, so zwar, dass oft dünne Späne von Sommer- und Herbstholz in einer staubigen, bloss aus zerfallendem Frühlingsholze entstandenen Masse zu liegen kommen. Wiesner überzeugte sich, dass diese Zerstörungsart des Holzes weder durch vegetabilische noch durch animalische Organismen bedingt ist, ja dass nicht einmal, wie dies beim Vergrauen sehr häufig der Fall ist, solche Organismen als gewöhnliche Begleiter dieser Zerstörungsart auftraten. Die Veränderungen, die das Holz bei der staubigen Vermoderung erleidet, sind rein physikalischer und chemischer Natur. Die Hygroskopicität eines staubig vermoderten Holzes ist grösser, als die jenes unveränderten Holzes, von dem ersteres abstammt (z. B. lufttrockenes Fichtenholz, unverändert verliert 10,503 Proc. Wasser, lufttrockenes staubigvermodertes Fichtenholz desselben Balkens 12,124 Proc. Wasser bei $110^{0} \mathrm{C}$.). Der Aschengehalt eines staubigvermoderten Holzes ist relativ grösser; als der des unveränderten Holzes (unverändertes Fichtenholz 0,269 Proc. Asche, in den ersten Stadien der staubigen Vermoderung begriffenen 0,554 Proc., vollständig vermoderten 2,568 Proc. Asche). Die Dichte staubig vermoderter Hölzer ist um ein Bedeutendes geringer als die des unveränderten Holzes (z. B. wie (0,295 bis 0,302:0,457).

Die chemischen Veränderungen, welche bei der staubigen Vermoderung auftreten, bestehen darin, dass die Zellmembranen sich nach und nach in Huminkörper umsetzen. Ulminsäure, Geïnsäure sind darin nachweisbar, aber weder Quellsäure noch Quellsatzsäure.

Die staubige Verwesung lässt sich durch Ausfüllung der an den Holzbalken entstehenden nach oben offenen Spalten mit einem schmelzenden Harze (Terpenthinharz, Pech) oder einem ähnlichen Körper hintanhalten. 
III. Die Bräunung der Hölzer.

Sie findet an Nadelhölzern (Föhren, Fichten, Tannen) statt. Das Holz wird oberflächlich rothbraun bis tief braun. Diese Bräunung ist eine Zerstörungsart solcher Hölzer, welche sich in einer vorwiegend feuchten Atmosphäre betinden, oder überhaupt reichlich befeuchtet werden. Sie kommt sowohl in waldreichen Gebirgsgegenden, als auch in ausgedehnten Auen der Niederungen vor. Die chemischen Umwandlungen bei der Bräunung bestehen in einer Umsetzung der Cellulose der Zellmembranen in Huminkörper, wodurch die Wände der Holzzellen bei Betrachtung unter dem Mikroskop eine gelbe bis lichtbraune Farbe erbalten. Die Umsetzung der Zellmembranen in Huminkörper schreitet vornehrnlich von a ussen nach innen vor. An Wasser geben solche Hölzer Qu ellsäure ab, an alkalische Flüssigkeit Ulminsäure und Geïnsäure; die darin unlöslichen Holzzellen geben mit concentrirter Schwefelsäure und Jod, mit Chlorzinkjodlösung und Kupferoxydammoniak die bekannten Zellstoffreactionen.

Die histologischen Veränderungen anlangend ist zu bemerken, dass aus den oberflächlichen Zellschichten die Intercellularsubstanz verschwunden ist. Die primären Membranen der Zellen werden zuerst zerstört und von hieraus schreitet die mechanische Zerstörung gegen die tertiäre Membran vor. Die Holzzellen fallen nicht nur der Dicke, sondern auch der Länge nach zertrümmert vom Holzkörper ab.

Bräunung and Vergrauung der Hölzer sind Zerstörungsarten, welche ihrem Wesen nach einzig und allein durch die atmosphärischen Wässer und Gase bedingt werden. Aber wie bei der Vergrauung so auch bei der Bräunung helfen die hinzukommenden Pilzsporen von innen her nach, das durch die Atmosphïrilien von aussen angegriffene $\mathrm{Holz}$ zu zertrümmern. (Wien. Sitzungsber. der k. k. Akad. der Wissensch. Mathem.naturw. Classe. Bd. XLIX. H.1. Abth. II. S.61-94.)

HI. Ludwig.

\section{Surrogate für Ebesholz und Elfenbein}

stellt man nach C. Chislain auf folgende Weise das. Zuvörderst wird aus Meeralgen durch dreistündiges Behandeln mit verdünnter Schwefelsäure, Eintrocknen und Zermahlen ein feines Pulver dargestellt. Von diesem 